\title{
DESENVOLVIMENTO DE MUDAS DE Moringa oleifera SUBMETIDAS A DIFERENTES SUBSTRATOS
}

\author{
Gleiciana Nascimento de Almeida1', Lunara Grazielly Costa da Silva1', Gleidiane Nascimento de Almeida', \\ Jenickson Rayron da Silva Costa², Maria José de Holanda Leite ${ }^{3}$, Elaine Cristina Alves da Silva ${ }^{4^{*}}$
}

${ }^{1}$ Graduada em Engenharia Florestal, Universidade Federal Rural do Semi Árido (UFERSA), Av. Francisco Mota, 572 - Bairro Costa e Silva, Mossoró/RN - CEP: $59.625-900$

${ }^{2}$ Graduando em Engenharia Florestal, Universidade Federal Rural do Semi Árido (UFERSA), Av. Francisco Mota, 572 - Bairro Costa e Silva, Mossoró/RN - CEP: 59.625-900

${ }^{3}$ Professora da Universidade Federal de Alagoas (UFAL), Centro de Ciências Agrárias de Alagoas (CECA), BR 104, Km 85, S/N, Mata do Rolo, Rio Largo/ AL - CEP: 57.100-000

${ }^{4}$ Professora da Universidade Federal Rural do Semi Árido (UFERSA), Centro de Ciências Agrárias, Av. Francisco Mota, 572 - Bairro Costa e Silva, Mossoró/RN - CEP: 59.625-900

*Autor para correspondência: Elaine Cristina Alves da Silva, elainemanancial@gmail.com

RESUMO: A Moringa oleifera é uma espécie arbórea que vem sendo utilizada como alternativa em diversas regiões como fonte de alimentação humana e animal, no tratamento de água, na produção de biodiesel, na indústria cosmética, na medicina tradicional e na indústria farmacêutica. $O$ objetivo foi avaliar o desenvolvimento de mudas de Moringa oleifera em diferentes substratos, sendo eles: solo do campus (T1), húmus de minhoca + esterco bovino (T2), esterco bovino + esterco de galinha (T3) e palha vegetal + areia + arisco (T4) com cinco repetições por tratamento. Semanalmente foi avaliado o número de folhas, diâmetro do colo e altura da planta, e ao final do experimento, medidos o comprimento da raiz e o peso seco da parte aérea e raiz, bem como o Índice de Dickson. Todas as variáveis foram afetadas significativamente em função dos diferentes substratos, sendo os maiores valores obtidos nas mudas que tiveram como substratos o T3 e T4. Os substratos que promoveram 0 melhor desenvolvimento das mudas de Moringa oleifera foi esterco bovino + esterco de galinha e palha vegetal + areia + arisco.

PALAVRAS CHAVE: Produção de mudas, moringa, Índice de Dickson.

\section{DEVELOPMENT OF CHANGES OF Moringa oleifera SUBMITTED TO DIFFERENT SUBSTRATES}

ABSTRACT: Moringa oleifera is an arboreal species that has been used as an alternative in several regions as a source of human and animal food, water treatment, biodiesel production, cosmetic industry, traditional medicine and the pharmaceutical industry. The objective was to evaluate the development of Moringa oleifera seedlings in different substrates. The substrates were tested: campus soil (T1), earthworm humus + bovine manure (T2), bovine manure + chicken manure (T3) and vegetal straw + sand + arisco (T4) with five replicates per treatment. Were evaluated weekly the number of leaves, neck diameter and height of the plant, and at the end of the experiment, the root length and dry weight of shoot and root were measured, as well as the Dickson Index. All variables were significantly affected as a function of the different substrates, being the highest values obtained in the T3 and T4 substrate. The substrates that promoted the best development of the Moringa oleifera seedlings was bovine manure + chicken manure and vegetal straw + sand + arisco.

KEY WORDS: Seedling production; moringa; Dickson Index.

\section{INTRODUÇÃO}

Pertencente à família Moringaceae, a Moringa oleifera é uma espécie arbórea originária do nordeste indiano, que é amplamente distribuída em vários países. Esta espécie de hábito arbustivo apresenta diversas aplicabilidades na medicina tradicional, na indústria de cosmética e como forragem animal. Também é bastante utilizada no tratamento de água tornando-a própria para o consumo humano (Ramachandran et al., 1980).

Por conta da alta concentração de cálcio, ferro, proteínas e aminoácidos a moringa tem se 
tornado um suplemento nutricional ideal, podendo ser considerada como uma Planta Alimentício não Convencional (PANC), da qual pode ser aproveitada desde suas folhas, frutos, flores e sementes. Também tem sido uma alternativa na elaboração de farinha para complementação alimentar no combate à desnutrição, especialmente em crianças e lactentes sendo utilizada como uma planta alimentícia (Teixeira, 2012).

A Moringa oleifera é uma espécie pouco exigente quanto à composição do substrato e adaptase a uma ampla faixa de solos, porém se desenvolve melhor em terra preta bem drenada ou em terra preta argilosa, preferindo um solo neutro a levemente ácido (Dalla Rosa, 1993; Oliveira, 2009).

0 conhecimento acerca da escolha do substrato ideal que promova um melhor desenvolvimento desta espécie é necessário, uma vez que, a produção de mudas de qualidade e em quantidade é uma das etapas essenciais para o estabelecimento dos povoamentos florestais. Diversos fatores são responsáveis para a obtenção de uma produção satisfatória, tais como temperatura, luz, umidade e substrato (Wendling, 2007).

Para a produção de mudas, o substrato possui a função de sustentar a planta e fornecer às raízes as condições necessárias para o seu crescimento e 0 estabelecimento, como a água, oxigênio e nutrientes.
Na escolha do substrato, é importante observar suas características físicas e químicas para a produção das mudas, tais como a composição nutricional, aeração, o pH e a condutividade. Além dos aspectos econômicos, como baixo custo e disponibilidade (Caldeira et al., 2008; Kratz e Weddling, 2013).

Sendo assim, este trabalho teve como objetivo avaliar o desenvolvimento de mudas de $M$. oleifera submetidas ao plantio em diferentes substratos.

\section{MATERIAL E MÉTODOS}

O experimento foi conduzido em casa de vegetação na Universidade Federal Rural do Semiárido (UFERSA), campus Mossoró- RN, no período de junho a agosto de 2017. Os frutos de moringa foram coletados em árvores localizadas no município Felipe Guerra $\mathrm{RN}$, estes foram secos naturalmente e as sementes retiradas dos frutos para posterior utilização.

Foi utilizado o delineamento inteiramente casualizado com quatro tipos de substratos: solo do campus (T1), húmus de minhoca + esterco bovino (T2), esterco bovino + esterco de galinha (T3) e palha vegetal +areia + arisco (1:1:1) (T4), com cinco mudas por tratamento, totalizando 20 unidades amostrais. A caracterização química dos substratos testados encontra-se na tabela 1.

Tabela 1. Caracterização química dos substratos de solo do campus (T1), húmus de minhoca + esterco bovino (T2), esterco bovino + esterco de galinha (T3) e palha vegetal +areia + arisco (T4) utilizados na produção de mudas de Moringa oleifera.

\begin{tabular}{|c|c|c|c|c|c|c|c|c|c|c|}
\hline Tratamento & $\begin{array}{l}\mathrm{pH} \\
\mathrm{H}_{2} \mathrm{O}\end{array}$ & $\begin{array}{c}\text { CE } \\
d S \cdot m^{-1}\end{array}$ & $\begin{array}{l}\text { M.O. } \\
\text { g.kg-1 }\end{array}$ & $\mathrm{P}$ & $\begin{array}{c}\mathrm{K}^{+} \\
\mathrm{mg} \cdot \mathrm{dm}^{-3}\end{array}$ & $\mathrm{Na}^{+}$ & $\mathrm{Ca}^{2+}$ & $\begin{array}{c}\mathrm{Mg}^{2+} \\
\text { Cmolc. } \mathrm{dm}^{-3}\end{array}$ & CTC & $\begin{array}{c}\text { PST } \\
\%\end{array}$ \\
\hline $\mathrm{T} 1$ & 8,93 & 0,15 & 0,52 & 73,6 & 69,1 & 90,6 & 3,10 & 0,30 & 3,97 & 10 \\
\hline T2 & 7,31 & 2,71 & 22,76 & 818,4 & 4476 & 714,5 & 26,5 & 22,15 & 11,39 & 36 \\
\hline T3 & 7,27 & 3,23 & 5,17 & 5,84 & 7109,9 & 422,9 & 19,3 & 26,4 & 63,21 & 5 \\
\hline T4 & 5,64 & 1,45 & 8,28 & 9,6 & 873,0 & 44,3 & 7,9 & 2,9 & 65,72 & 3 \\
\hline
\end{tabular}

CE: condutividade elétrica, M.O.: matéria orgânica, CTC: Capacidade de troca catiônica, PST: percentagem de sódio trocável.

As sementes foram semeadas em solo do substrato T1, disposto em bandejas de plásticos, a uma profundidade de um $\mathrm{cm}$. Após 15 dias da emergência, as mudas foram repicadas e acondicionadas em sacos plásticos com dimensões $16 \times 22 \mathrm{~cm}$, preenchidos com os substratos selecionados as quais permaneceram por 35 dias. Em seguida, foi realizada uma marcação no caule a um cm do solo, com um marcador permanente, para as análises da altura e diâmetro.
Semanalmente, as mudas foram avaliadas quanto ao número de folhas (NF), através da contagem; a altura da plântula $(H)$, com auxílio de uma régua graduada a partir da marcação no caule até o ápice da gema apical; e o diâmetro do colo obtido com um paquímetro digital.

Ao final do experimento, foi medido o sistema radicular, pelo comprimento da raiz principal (RP) com 0 auxílio de uma régua e em seguida, as mudas foram 
separadas em parte aérea e raiz, colocadas em sacos de papel, levadas para estufa regulada a $65^{\circ} \mathrm{C}$ até obtenção do peso constante (72 horas) e, após este período, foi utilizada balança analítica de precisão de 0,001 g para a obtenção do peso da matéria seca da parte aérea (PMSPA) e da raiz (PMSR).

Foi determinado 0 índice de qualidade de Dickson (IQD) em função da altura da parte aérea $(H)$, do diâmetro do colo (DC), do peso de matéria seca da parte aérea (PMSPA) e do peso de matéria seca das raízes (PMSR), por meio da fórmula de IQD = PMST/ [(H/DC)+(PMSPA/PMSR)] (Dickson et al., 1960).

Os dados obtidos foram submetidos à análise de variância e as médias comparadas pelo teste de
Tukey ao nível de $5 \%$ de probabilidade, utilizando o programa estatístico Assistat.

\section{RESULTADOS E DISCUSSÕES}

Observou-se que a altura das mudas foi afetada significativamente a partir da terceira semana, em que o T2 apresentou uma diferença de $31 \%$ em relação T4 e ao final do período avaliado a redução foi de $42 \%$. As mudas que se desenvolveram no substrato $\mathrm{T} 4$, triplicaram sua altura enquanto no tratamento T2 e T3, aumentaram apenas $25 \%$ em relação aos seus valores iniciais (Figura 1).

Figura 1- Altura de mudas de Moringa oleifera sob diferentes substratos.

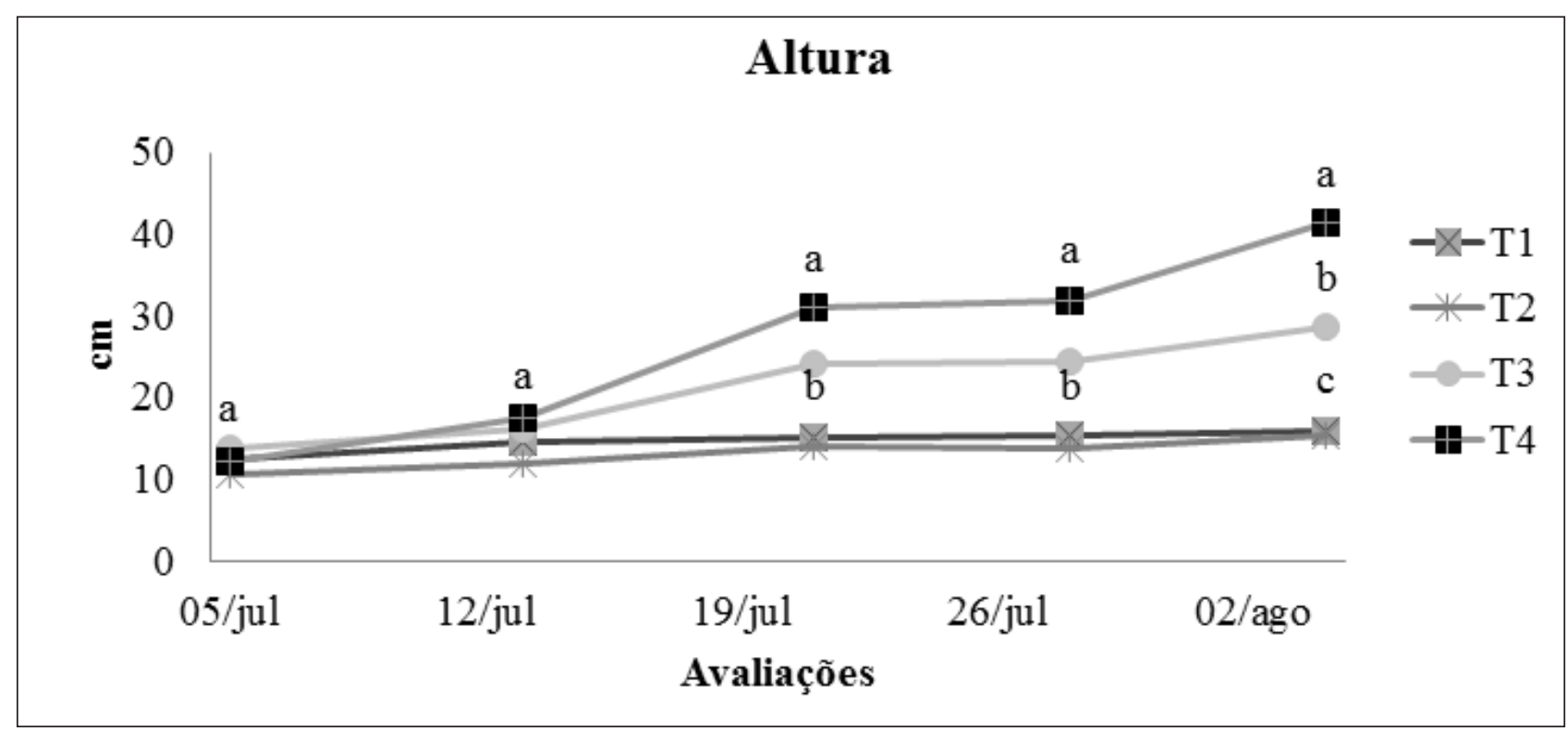

T1- solo da UFERSA, T2 - húmus de minhoca + esterco bovino, T3 - esterco bovino + esterco de galinha, T4 - palha vegetal +areia + arisco.

Embora o substrato T2 tenha apresentado o maior valor de matéria orgânica (22,76 g.kg-1), as plantas que se desenvolveram no mesmo, tiveram a menor altura $(11,5 \mathrm{~cm})$, enquanto as plantas do substrato T4 (8,28 g.kg-1 de M.O.) foi de 41,25 cm. De acordo com Santos et al (2016), a matéria orgânica ajuda fornecer uma maior retenção de água e nutrientes 0 que favorece um maior incremento das mudas. 0 substrato 4 apresentou os menores valores de fósforo, potássio, sódio, cálcio e magnésio, também uma menor condutividade elétrica e pH, entretanto, apresentou uma maior CTC (Tabela1).

Resultados semelhantes foram encontrados por Camargo (2011), que ao avaliar o efeito de substratos com diferentes composições e concentração de matéria orgânica, também observaram que o substrato húmus de minhoca mesmo adicionada matéria orgânica não houve aumento em altura nas mudas de M. oleifera. Neves et al. (2010) avaliando o uso de substratos alternativos para produção de mudas de moringa, concluíram que o substrato esterco de aves proporcionou um maior crescimento em altura.

Para o diâmetro, os maiores valores foram observados para os substratos T3 e T4 (11,25 mm), os quais a partir da segunda semana apresentaram diferenças estatísticas dos demais e aumentou 1,5x em relação à primeira avaliação destes mesmos tratamentos (Figura 2). 
Figura 2- Diâmetro de mudas de Moringa oleifera sob diferentes substratos.

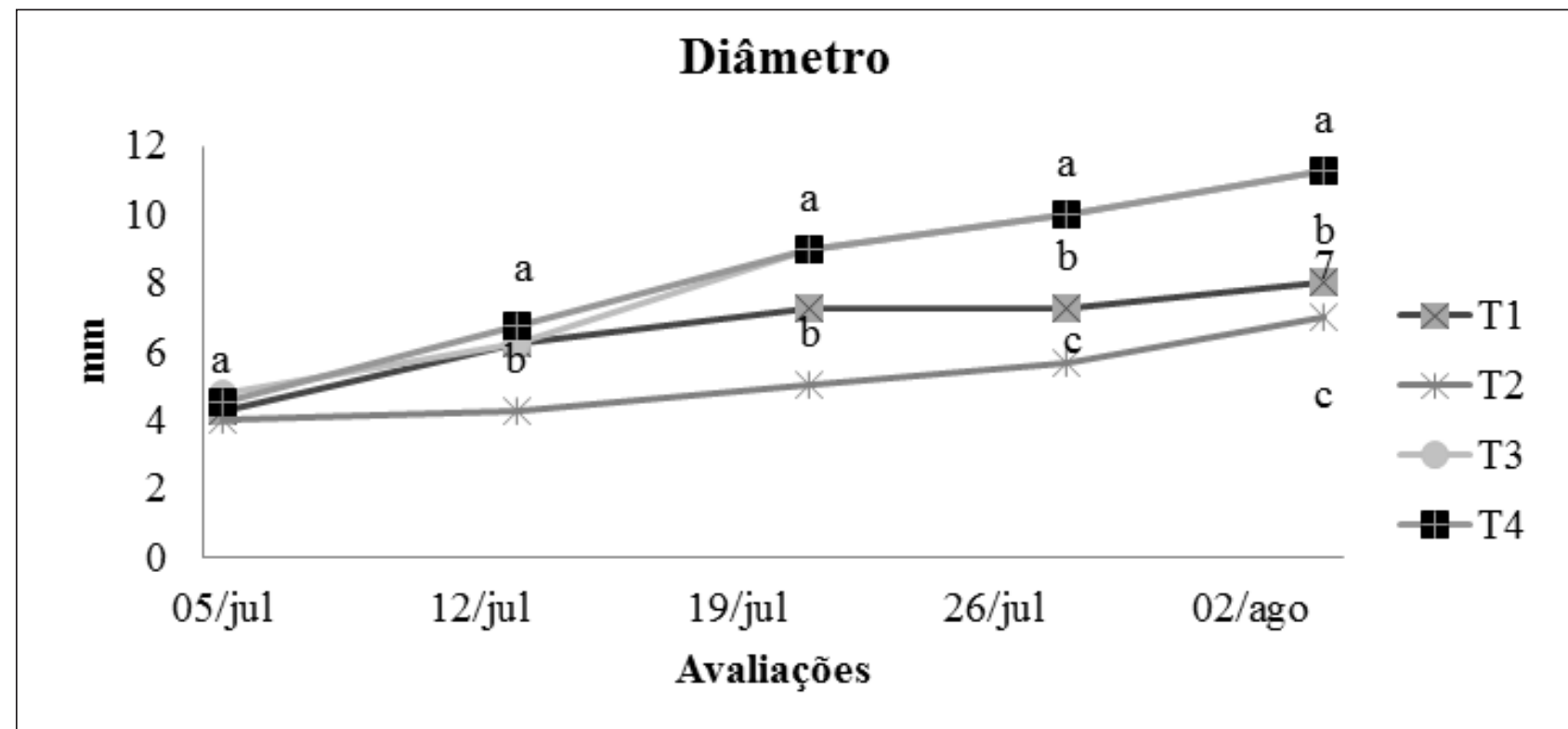

T1- solo da UFERSA, T2 - húmus de minhoca + esterco bovino, T3 - esterco bovino + esterco de galinha, T4 - palha vegetal +areia + arisco.

Com relação ao número de folhas, os maior emissão de folhas (6), enquanto os substratos tratamentos T3 e T4 apresentaram resultados T1 e T2 reduziram 27\%, em relação aos primeiros estatísticos semelhantes, os quais proporcionaram uma tratamentos citados (Figura 3).

Figura 3- Número de folhas de mudas de Moringa oleifera sob diferentes substratos.

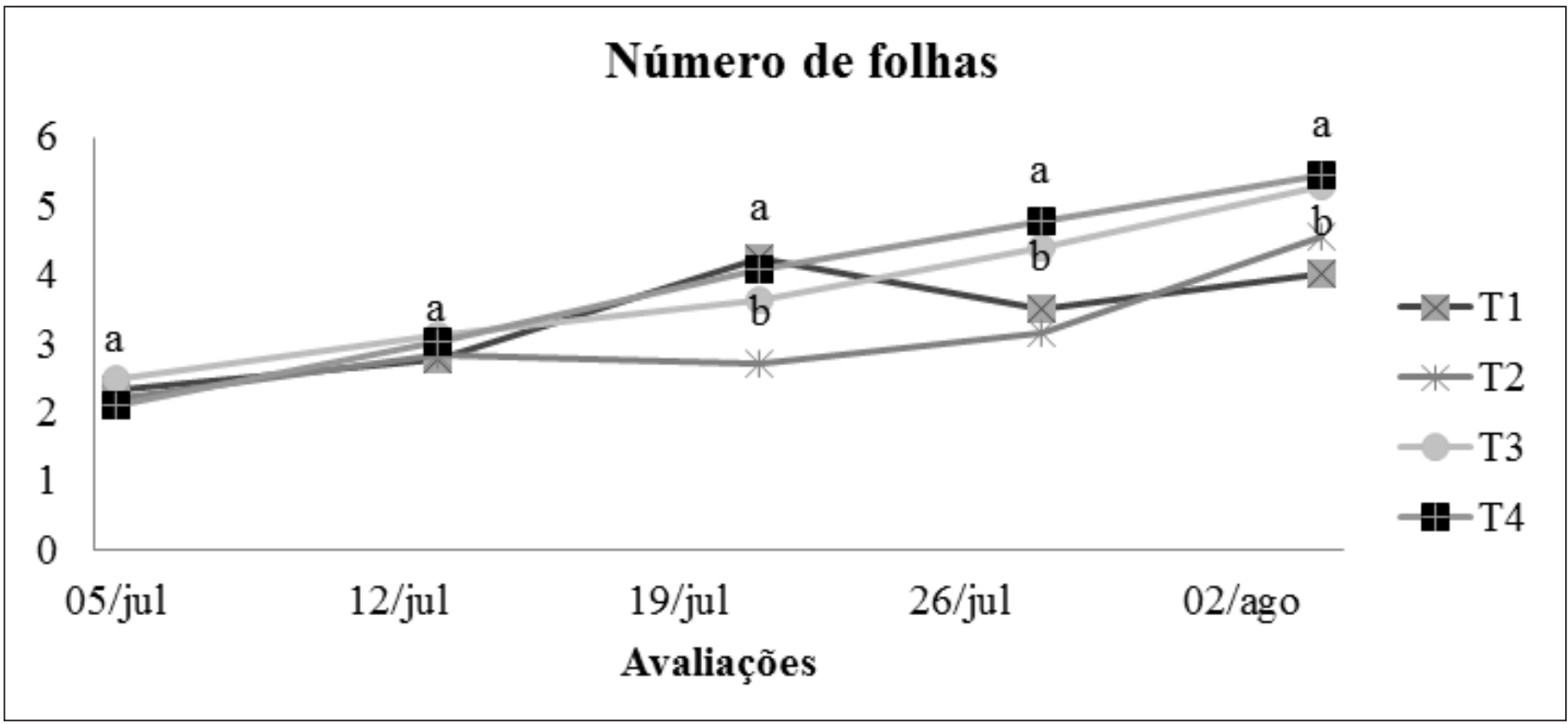

T1- solo da UFERSA, T2 - húmus de minhoca + esterco bovino, T3 - esterco bovino + esterco de galinha, T4 - palha vegetal +areia + arisco.

Medeiros et al. (2010) ao avaliarem diferentes fontes de matéria orgânica na produção de mudas, constatou que as plantas de pinhãomanso apresentaram maior altura, além do maior número de folhas e área foliar, nos tratamentos que continham esterco bovino quando comparado aquelas produzidas em substrato contendo cama de frango.

Araujo et al. (2013) ao testarem diferentes substratos orgânicos na produção de mudas de mamoeiro Formosa, verificou que o substrato que continha húmus de minhoca proporcionou os maiores 
valores para altura das plantas, números de folhas e diâmetro caulinar e massa seca da raiz, já o esterco bovino proporcionou maior comprimento da raiz.

Outra variável importante para a qualidade da muda é o comprimento do sistema radicular.
Todavia, observou-se que, o T4 outra vez obteve os maiores valores com relação ao comprimento da raiz, enquanto o $\mathrm{T} 2$ reduziu $41 \%$ em relação ao T4 (Tabela 2).

Tabela 2- Valores médios do comprimento da raiz (CR), peso seco da parte aérea (PSPA), peso seco da raiz (PSR) e índice de Dickson (IDQ) de mudas de Moringa oleifera sob diferentes substratos.

\begin{tabular}{ccccc}
\hline Tratamentos & CR $(\mathrm{cm})$ & PSPA $(\mathrm{g})$ & PSR $(\mathrm{g})$ & IQD \\
\hline T1 & $11,5 \mathrm{~b}$ & $0,81 \mathrm{bc}$ & $1,96 \mathrm{a}$ & $0,53 \mathrm{a}$ \\
T2 & $9,6 \mathrm{~b}$ & $0,63 \mathrm{c}$ & $0,18 \mathrm{~b}$ & $0,12 \mathrm{~b}$ \\
T3 & $13 \mathrm{ab}$ & $1,65 \mathrm{ab}$ & $1,45 \mathrm{a}$ & $0,57 \mathrm{a}$ \\
T4 & $16,3 \mathrm{a}$ & $1,74 \mathrm{a}$ & $1,73 \mathrm{a}$ & $0,52 \mathrm{a}$ \\
\hline
\end{tabular}

T1 - solo do campus, T2 - húmus de minhoca + esterco bovino, T3 - esterco bovino + esterco de galinha, T4 - palha vegetal +areia + arisco. ${ }^{*}$ Letras iguais na coluna não diferem entre si estatisticamente ( $5 \%$ de probabilidade).

0 peso seco da parte aérea reduziu $64 \%$ para o T2, em relação ao maior resultado que foi encontrado para o T4. Este mesmo comportamento foi observado para o peso seco das raízes, no entanto com a redução mais acentuada de $90 \%$ (Tabela 2). 0 peso de matéria seca da parte aérea é um bom indicador da capacidade de resistência das mudas às condições adversas após o plantio (Nóbrega, 2010).

Ao testarem diferentes substratos para a formação de mudas de meloeiro, onde o substrato (pó de fibra de coco seco + húmus de minhoca (1:1), Bezerra et al. (2001) obtiveram o melhor resultado para o peso seco da parte aérea.

Ensinas et al. (2011) observaram que os tratamentos com a maior concentração do substrato comercial $(100 \%$ substrato e $80 \%$ substrato $+20 \%$ húmus) apresentaram os maiores pesos de matéria seca (0,54 e 0,44 g, respectivamente), quando avaliou o desenvolvimento de mudas de rúcula utilizando-se diferentes combinações de substrato.

Os resultados obtidos na presente pesquisa podem estar relacionados à granulometria do substrato, permitindo maior espaço poroso, possibilitando um maior crescimento e influenciando na aeração das raízes (Tavares Júnior, 2004).

Com relação ao Índice de qualidade de Dickson (IQD), o melhor resultado foi verificado no T1, T3 e $\mathrm{T} 4$, os quais não diferiram estatisticamente entre si. 0 substrato T2 resultou no menor valor de IQD (Tabela 2). Segundo Caldeira et. al., (2012) quanto maior o IQD, melhor é a qualidade da muda produzida. Esse índice considera a interação entre vários fatores resultando na determinação da qualidade das mudas (Binotto, 2007).

De acordo com Favalessa (2011), o índice de qualidade de Dickson pode variar em função de diversos fatores como a espécie, manejo das mudas no viveiro, tipo e proporção do substrato, volume do recipiente e, principalmente, de acordo com a idade em que muda foi avaliada.

Os substratos esterco bovino + esterco de galinha (T3) e palha vegetal + areia + arisco (T4) proporcionaram os melhores resultados em todas as variáveis avaliadas, no entanto, o T4 foi o substrato de menor custo, em relação aos demais, exceto ao substrato solo do campus da universidade, o qual não teve custo. 0 substrato $\mathrm{T} 4$ foi produzido a partir de uma mistura de palha de carnaúba, espécie abundante na região do Rio Grande do Norte, que juntamente com a areia e 0 arisco o torna um substrato interessante para a produção de moringa.

\section{REFERÊNCIAS}

Araújo, A. C.; Araújo, A. C.; Dantas, M. K. L.; Pereira, W. E.; Aloufa, M. A. I. Utilização de substratos orgânicos na produção de mudas de mamoeiro Formosa. Revista Brasileira de Agroecologia, 2013, 8, 1, 110-116.

Bezerra, A. M. E.; Momenté, V. G.; Medeiros Filho, S.; Germinação de sementes e desenvolvimento de plântulas de moringa (Moringa oleifera Lam.) em função do peso da semente e do tipo de Substrato. Horticultura Brasileira, 2004, 22, 2, 295-299. 
Bezerra, F. C.; Bezerra, G. S. S.; Diferentes substratos para a formação de mudas de meloeiro. Horticultura Brasileira, 2001, 19, suplemento CD-ROM.

Binotto, A. F. Relação entre variáveis de crescimento e o Índice de Qualidade de Dickson em mudas de Eucalyptus grandis W. Hill ex Maind e Pinus elliottii var. elliottii - Engelm. 2007. 53 f. Dissertação (Mestrado em Engenharia Florestal) - Universidade Federal de Santa Maria, Santa Maria, 2007.

Camargo, R., Substratos para produção de mudas de Moringa oleifera I. em bandejas. Agropecuária Técnica, 2011, 32, 1, 72-78.

Caldeira, M. V. W.; Delarmelina, W. M.; Lübe, S. G.; Gomes, D. R.; Gonçalves, E. O.; Alves, A. F. Biossólido na composição de substrato para a produção de mudas de Tectona grandis. Floresta, 2012, 42, 1, 77 - 84.

Dalla Rosa, K. R. Moringa oleifera: a perfect tree forhome gardens. Hawai: NFTA, Agroforestry Species Highlights, 1993, 1, 2p.

Dickson, A.; Leaf, A. L.; Hosner, J. F. Quality appraisal of white spruce and white pine seedling stock in nurseries. The Forest Chronicle, 1960, 36, 10-13.

Ensinas, S. C.; Maekawa Junior, M. T., Cândido, B. Desenvolvimento de mudas de rúcula em diferentes combinações de substrato. Revista Científica Eletrônica de Agronomia, 2011, 18, 1, 1-7.

Favalessa, M.; Substratos renováveis e não renováveis na produção de mudas de Acacia mangium, 2011, 60 p. Monografia (Engenharia Florestal) -Universidade Federal do Espirito Santo. 2011.

Kratz, D.; Wendling, I.; Produção de mudas de Eucalyptus Dunni em substratos renováveis. Revista Floresta, 2013, 43, 1, 125 -136.

Mederiros, K. A. A. L., Safiatti, V., Silva, H., Lima, R., Lucena, A. M. A., Vasconcelos, G. C., Arriel, N. H. C.; Mudas de pinhão manso (Jatropha curcas I.) produzidas em diferentes fontes e doses de matéria orgânica. IV Congresso Brasileiro de Mamona e I Simpósio Internacional de Oleaginosas Energéticas, João Pessoa, PB - 2010.
Neves, J. M.; Silva, H. P.; Duarte, R. F.; Uso de substratos alternativos para produção de mudas de moringa. Revista Verde, 2010, 5, 1, 173 - 177.

Nobrega, R. S. A.; Ferrreira, P. A. A.; Santos, J. G. D.; Boas, R. C. V.; Nóbrega, J. C. A.; Moreira, F. M. S. Substratos para o crescimento de mudas de Trema micrantha (L.) Blumes. Revista Brasileira de Ciências Agrárias, 2010, 5, 3, 392-397.

Oliveira, F. R. A.; Oliveira, F. A.; Guimaraes, P. I.; Medeiros, J. F.; Oliveira, M. K. T.; Freitas, A. V. L.; Medeiros, M. A.; Emergência de plântulas de moringa irrigada com água de diferentes níveis de salinidade. Bioscience Journal, 2009, 25, 5, 66-74.

Ramachandran, C.; Peter, K. V.; Gopalakrishnan, P. K. Drumstick (Moringa oleifera) a multipurpose Indian vegetable. Economy Botany, 1980, 34, 276-283.

Santos, P. L. F.; Castilho, R. M. M.; Atributos físicos de diferentes substratos para fins de desenvolvimento de plantas. Simpósio nacional de tecnologia em agronegócio, Jales/SP, 2016.

Tavares Junior, J. E. Volume e granulometria do substrato na formação de mudas de café. 2004. 59 p. Dissertação (Mestrado em fitotecnia) - Universidade de São Paulo. 2004.

Teixeira, E. M. B. Caracterização química e nutricional da folha de Moringa (Moringa oleifera Lam.). 2012. f. 94. Tese de Doutorado. Araraquara, São Paulo. 2012.

Wendling, I.; Guastala, D.; Dedecek, R.; Características físicas e químicas de substratos para produção de mudas de llex Paraguariensis st. Hil. Revista Árvore, 2007, 31, 2, 209-220. 